\title{
CHANGES IN EPILEPTOGENICITY BIOMARKERS AFTER STEREOTACTIC- THERMOCOAGULATION
}

Margherita Contento $\mathrm{MD}^{1}$, Francesca Pizzo, $\mathrm{MD}, \mathrm{PhD}^{2-3}$, Víctor J. López-Madrona $\mathrm{PhD}^{2}$, Stanislas Lagarde MD,PhD ${ }^{2-3}$, Julia Scholly MD, $\mathrm{PhD}^{3,6}$, Agnes Trébuchon MD,PhD ${ }^{2-3}$, Samuel Medina Villalon Eng ${ }^{2-3}$, Bernard Giusiano, MD, $\mathrm{Phd}^{2-3}$, Didier Scavarda MD, $\mathrm{PhD}^{4}$, Romain Carron MD, $\mathrm{PhD}^{5}$, Nicolas Roehri $\mathrm{PhD}^{2}$, Christian G. Bénar $\mathrm{PhD}^{2}$, Fabrice Bartolomei MD, $\mathrm{PhD}^{2-3}$

1 Department of Neurosciences, Drug Research, and Child's Health, University of Florence, Florence, Italy.

2 Aix Marseille Univ, INSERM, INS, Inst Neurosci Syst, Marseille, 13005, France.

3 APHM, Timone Hospital, Epileptology and cerebral rhythmology, Marseille, 13005, France.

4 APHM, Timone Hospital, Pediatric Neurosurgery Department, Marseille, France

5 APHM, Timone Hospital, Stereotactic and Functional Neurosurgery, Marseille, France.

6 Aix Marseille Univ, CEMEREM, UMR 7339, Hôpital Timone, Marseille, France

Corresponding authors: Dr Francesca Pizzo and Prof Fabrice Bartolomei, MD, PhD Epileptology Department, CHU Timone, 264 Rue Saint-Pierre, 13005, Marseille, France Tel: +33491385833 Fax: +33491385826 Email: fabrice.bartolomei@ap-hm.fr

Keywords for use by abstracting services: Thermocoagulation, stereo-electroencephalography, epileptogenic biomarkers.

number of text pages: 13

number of words:4073 
number of references: 33

number of figures: 4

number of tables: 2

ORCID number for the first author: https://orcid.org/0000-0001-8806-6685

ORCID number for the senior author: https://orcid.org/0000-0002-1678-0297

ORCID numbers for the authors designated as corresponding: https://orcid.org/0000-0001-63451288, https://orcid.org/0000-0002-1678-0297

\section{ABSTRACT}

Objective: Stereo-electroencephalography-guided radiofrequency thermocoagulation (SEEG-guided RF-TC) aims at modifying epileptogenic networks to reduce seizure frequency. High-frequency oscillations (HFOs), spikes, and cross-rate are quantifiable epileptogenic biomarkers. In this study, we sought to evaluate, using SEEG signals recorded before and after thermocoagulation, whether a variation in these markers is related to the therapeutic effect of this procedure and to the resective surgery outcome.

Methods: Interictal segments of SEEG signals were analyzed in 38 patients during presurgical evaluation. We used an automatized method to quantify the rates of spikes, HFOs, and cross-rate (a measure combining spikes and HFOs) before and after thermocoagulation. We analyzed the differences both at an individual level with a surrogate approach and at a group level with an analysis of variance. We then evaluated the correlation between these variations and the clinical response to RF-TC and to subsequent resective surgery.

Results: After thermocoagulation, 19 patients showed a clinical improvement. At the individual level, clinically improved patients had more frequently a reduction in spikes and cross-rate in the 
epileptogenic zone than patients without clinical improvement $(\mathrm{p}=0.002 ; \mathrm{p}=0.02)$. At a group level, there was a greater decrease of HFOs in epileptogenic and thermocoagulated zones in patients with clinical improvement $(\mathrm{p}<0.05)$ compared to those with no clinical benefit. Eventually, a significant decrease of all the markers after RF-TC was found in patients with a favorable outcome of resective surgery (spikes: $\mathrm{p}=0.026$; HFOs: $\mathrm{p}=0.03$; cross-rate: $\mathrm{p}=0.03$ ).

Significances: Quantified changes in the rates of spikes, HFOs, and cross-rate can be observed after thermocoagulation and the reduction of these markers correlates with a favorable clinical outcome after RF-TC and with a successful resective surgery. This may suggest that interictal biomarkers modifications after RF-TC can be clinically used to predict the effectiveness of the thermocoagulation procedure and the outcome of resective surgery.

KEYWORDS FOR USE BY ABSTRACTING SERVICES: Thermocoagulation, Stereoelectroencephalography, Epileptogenic biomarkers.

\section{KEY POINTS BOX}

1) Quantified changes in the rates of spikes, high-frequency oscillations, and cross-rate were observed after thermocoagulation with an automatized method.

2) The reduction of these markers after thermocoagulation correlates with a favorable clinical outcome after this procedure.

3) The reduction of all markers appeared to be related to a favorable outcome of resective surgery, regardless of the clinical improvement after thermocoagulation.

4) Interictal biomarkers modifications after thermocoagulation may represent a clinical predictor of the effectiveness of the procedure. 


\section{INTRODUCTION}

SEEG-guided RF-TC aims to "alter" epileptogenic networks in patients with focal drug-resistant epilepsy. ${ }^{1,2,3,4}$ It is performed at the end of the SEEG recordings employing the same intracerebral electrodes to generate thermocoagulative lesions in the areas of interest. ${ }^{1,2,3,4,5,6}$ The clinical improvement rate at 12 months, usually temporary, is between $28-48 \% .^{1,5,7}$ Clinical predictors of a good outcome are: malformations of cortical development (MCD, particularly nodular heterotopias), number of intra-lesional RF-TC sites, occipital location of the RF-TC targets, and the presence of a visible lesion on pre-operative MRI. ${ }^{5,6,7,8,9,10,11}$ Previous research showed that a focal low-voltage high-frequency activity at seizure onset and a lowered epileptogenic threshold at stimulations in the coagulated areas could be positive predictors of response to thermocoagulation as well as the presence of continuous or sub-continuous interictal epileptiform discharges (SCIEDs) in pre-RF-TC SEEG traces. ${ }^{6,12}$ Three studies observed the effects of RF-TC on spikes using a visual analysis showing a voltage reduction and/or a decreased frequency of interictal spikes immediately after RFTC. However, these modifications were not significantly associated with clinical response. ${ }^{6,12,13}$ A case report from our group showed a quantitative reduction of interictal high-frequency oscillations (HFOs) and spikes after RF-TC and its correlation with a significantly improved seizure control. ${ }^{14}$ The aim of the present study was to perform a quantitative and reproducible assessment of the effects of RF-TC on the electrophysiological markers of epileptogenicity (spikes, HFOs, and cross-rate) ${ }^{15,16}$ and to study the correlation between these variations and the clinical response.

\section{METHODS}

\section{$\underline{\text { 2.1 Patients }}$}

We extracted from the database of the Epileptology Unit of La Timone Hospital all the patients in the period 01/2013-12/2017 according to the following inclusion criteria: having undergone SEEGguided RF-TC for drug-resistant epilepsy and the availability of at least 15 minutes of SEEG recordings just before and after the end of RF-TC session sampled at least at $1024 \mathrm{~Hz}$. 
The institutional review board approved this study (C3BHV2) and written patient consent was obtained both for the implantation of the electrodes for SEEG and the subsequent RF-TC procedures. Patients underwent phase I pre-surgical non-invasive exams (long-term scalp video-EEG monitoring, neuropsychological testing, high-resolution MRI, 18-FDG-PET). Video-SEEG recording was necessary for a reliable localization of the epileptogenic zone (EZ) and consequently of the surgical target. $^{17}$ At the end of the SEEG exploration, SEEG-guided RF-TC was performed and the intracerebral activity was recorded before, during, and at the end of the procedure. Seizures frequency and possible adverse events of the RF-TC were collected at the first follow-up visit performed one month after the procedure. Regarding the clinical effects of RF-TC, we defined the patients as clinically improved when they showed either at least a 50\% decrease in seizure frequency or seizure freedom. These improvements to be considered adequate should have lasted for a minimum of one month for patients with daily seizures before RF-TC, for a minimum of two months for patients with weekly seizures, and for a minimum of 6 months for patients with monthly seizures. When epilepsy resective surgery was performed, the day before surgery was considered as the last post-RF-TC follow-up time. The outcome of resective surgery was assessed using Engel's classification at least 12 months after the procedure. ${ }^{18}$

\subsection{SEEG and thermocoagulation procedures}

Intracerebral multiple contact electrodes (Alcis, Besançon, France or Dixi Microtechniques, Besançon, France; $10-15$ contacts, length: $2 \mathrm{~mm}$, diameter: $0.8 \mathrm{~mm}, 1.5 \mathrm{~mm}$ apart) were implanted for SEEG exploration according to Talairach's stereotactic method. ${ }^{17,19,20}$ The anatomical targets of electrodes were decided according to the hypotheses about EZ localization coming from phase 1. A postoperative CT scan and/or MRI were performed to verify the absence of any complication and the spatial accuracy of the implantation. CT-scan/MRI data coregistration was performed to check the anatomical location of each contact along the electrode's trajectory. For this purpose, we used the open-source software GARDEL. ${ }^{21}$ Video-SEEG recordings were performed as long as necessary (1 
to 3 weeks) to record several of the patient's habitual seizures. The contacts used for RF-TC were selected for each patient according to the following criteria: (1) contacts sampling structures that belong to the EZ, as defined by visual analysis complemented by quantitative SEEG-signal analysis using the Epileptogenicity Index ${ }^{22}$ (2) contacts sampling structures that belong to the early propagation zone (3) contacts located within or at the MRI-visible borders of the lesion suspected to be epileptogenic, and/or (4) induction of habitual ictal clinical phenomena by electrical stimulation of those contacts. ${ }^{23}$ If the selected contacts sampled a functionally critical area or if they were in proximity of vascular structures, they were excluded from the RF-TC. The RF-TC lesions were produced between 2 contiguous contacts of the selected electrodes connected to a radiofrequency lesion generator equipment (Neuro N50 and Neuro N100 INOMED, Germany with Dixi Medical and Alcis electrodes, respectively) as in previous studies. ${ }^{7,14}$ No anesthesia was employed and the procedures were done under video-SEEG monitoring. Repeated successive RF-TC procedures were performed if the impedances of the contacts used for bipolar RF-TC did not significantly decrease after RF-TC (i.e., an effective necrosis was still not completely achieved). If focal seizures occurred during RF-TC, the procedure was interrupted and was resumed a few minutes after.

\subsection{SEEG signal Analysis}

SEEG-signal was recorded on a digital system (Natus Medical Incorporated Pleasanton, California, USA) with a sampling at least at $1024 \mathrm{~Hz}$ with 16 bits resolution, a hardware high-pass filter (cut-off=0.16 Hz), and an antialiasing low-pass filter (cut-off=340 Hz). In order to avoid potential confounding factors and for comparison purposes, we decided to analyze the 15-minute time frame just before the beginning of the RF-TC session and the 15-minute time frame immediately after that the last RF-TC has been performed. In our center, thermocoagulation procedure is done under SEEG monitoring that allows continuous recordings of interictal activity before, during, and immediately after the treatment. All patients were awake during these periods of time. Signal analysis was computed using AnyWave software. ${ }^{24}$ A specific montage was created for each patient removing the channels with too many artifacts (including in some cases thermocoagulated contacts). Moreover, on 
the remaining channels, a visual evaluation of the traces before the analysis was performed to identify and remove the sections with artifacts. Spikes and HFOs (80-330 Hz) were automatically quantified in AnyWave using Delphos (Detector of ElectroPhysiological Oscillations and Spikes). ${ }^{25}$ The method applied by Delphos has been explained elsewhere. ${ }^{14,25}$

\section{$\underline{\text { 2.4 Analyzed Variables }}$}

We calculated the rates (number of events/minute) before and after RF-TC of the following markers: spikes, HFOs, and cross-rate. The cross-rate corresponds to the geometrical mean of the spikes and HFOs rates obtained by calculating the square root of the product of the two rates. ${ }^{16}$ Rates were first calculated from all channels and, secondarily divided into three categories of interest as identified by an epileptologist during SEEG evaluation. Firstly, the irritative zone is defined as the channels presenting, all along SEEG recordings, interictal activity. Secondly, the EZ is considered as the channels involved at seizure onset which is defined by quantitative SEEG-signal analysis using the Epileptogenicity Index ${ }^{22}$ and by visual analysis. Finally, the thermocoagulated zone is composed of the thermocoagulated channels. In some patients, these regions could be overlapped. The following clinical variables were collected: sex, age at seizure onset, age at RF-TC, duration of epilepsy, seizure frequency, presence of a lesion on preoperative MRI, etiology of epilepsy (assessed by MRI or histology), topography of epilepsy (temporal, posterior, insular, frontal, multilobar), anatomic extent of epileptogenic, irritative, and thermocoagulated zones (classified as regional if limited to a sublobar involvement, or extended if the entire lobe or several lobes were involved ${ }^{6}$ ). The presence of SCIEDs in the SEEG traces preceding RF-TC was independently evaluated by two clinicians.

\section{$\underline{2.5 \text { Statistics }}$}

All the analyses were done with custom code in MatLab® (MathWorks, Natick, MA, USA) and GraphPad Prism v7.00 (GraphPad Software, La Jolla California USA). Unless otherwise stated, pvalue was considered significant at 0.05 . 


\subsubsection{Intra-individual statistics}

To test the effect of RF-TC on each marker in each area and in each patient individually we followed a surrogate approach (nonparametric permutation test). ${ }^{26}$ The general idea of this methodology is to generate simulated data with the same properties as the original one, but removing the condition to test, namely the RF-TC. First, we combined the events across channels into a single time-series. Then, each surrogate dataset was modeled by randomly shuffling the time intervals between markers. Therefore, each dataset has the same number of markers as the original one. The simulated dataset was then split into two windows with the same duration, which represent the epochs before and after RF-TC, and the rate difference was computed. As all the events had been randomly distributed into the two time-windows, the difference between both conditions would be by chance, reflecting the variability of the data without the effect of the RF-TC. This process was repeated 5000 times, obtaining the same number of surrogate results. The distribution of these values approximates a Normal distribution. The original value, i.e. the rate between post minus pre in the combined timeseries, can be directly compared with this distribution and its distance from the mean of the distribution (in terms of standard deviations) would indicate the probability that the thermocoagulation influences the marker rate. Finally, the associated p-value was defined as the previous normal cumulative distribution function, evaluated at the original result, i.e., the area under the curve of the distribution (see Fig 1A, B, C). Eventually, among those with a significant intraindividual reduction at the surrogate analysis, the proportion of clinically improved versus not clinically improved patients after RF-TC was tested by Fisher's Exact Tests. Also the proportion of clinically improved versus not clinically improved patients after resective surgery was tested by Fisher's Exact Tests among those who performed resective surgery and had a significant intraindividual reduction at the surrogate analysis. 


\subsubsection{Analysis at a group level}

The method described above based on intra-individual statistics is limited when inferring group changes, as the variance of the data in each case is lost. To further test the effect of RF-TC, we performed a group analysis of the variance of the marker rates.

The rate of each marker was averaged between all the available channels for each patient and then, to facilitate the statistical analyses, we used the difference of the "rate after" minus the "rate before" RF-TC, whose distribution approximates a Normal distribution. Unless otherwise stated, we used a ttest to compare whether the differences post-pre were different from zero, and a two-way ANOVA followed by Holm-Sidak correction to compare the differences between several conditions.

First, we have analyzed if the RF-TC produces a decrease in the frequency of the different markers in all the recorded channels regardless of the clinical outcome of the patients: we compared if the difference between post and pre values of the rates of the markers were lower than zero with a onesample t-test. After that, we tested if the effect of RF-TC was region-specific repeating the analysis in each area of interest (irritative, epileptogenic, and thermocoagulated zone; t-test).

As not all patients presented a clinical improvement after RF-TC, we asked whether this factor was related to the variation in the marker rate. Therefore, we divided the patients into two groups according to the clinical outcome and compared the difference between post and pre values of the rates of the markers in each area of interest with a two-way ANOVA followed by Holm-Sidak correction.

As many factors may influence the markers, we have computed a multiple linear regression model including them as predictors of the difference between post and pre values of the rates of the markers. The joint analysis of several features as predictors of the difference in the rate of each marker was done using multiple linear regressions (function regress.m in Matlab). First, a single model is fitted using each predictor multiplied by a beta factor. The contribution of one specific predictor to the total explained variance of the model is estimated by fitting a reduced multiple linear regression without that predictor and computing the difference between variances in the full model 
minus the reduced one. This analysis allows an estimation of the contribution of each variable that cannot be accounted by any other variable in the model. The statistical significance of each factor was tested with a t-statistic of the hypothesis that the corresponding coefficient is equal to zero or not.

Finally, to analyze if the effect of the RF-TC on the markers may be a predictor of the success of the resective surgery, we have compared the difference between post and pre values of all channels in patients with a successful outcome of surgery versus those with a negative outcome, independently of any other factor (two-way ANOVA followed by Holm-Sidak correction).

\section{RESULTS}

\section{$\underline{\text { 3.1 Population features, thermocoagulation procedure, clinical response, and surgery }}$}

After applying the selection criteria, 38 patients were evaluated (21 men and 17 women). The median age at the time of RF-TC was 19 years old (1-61) and the median age of onset of epilepsy was 5.5 years old (0-36) with a median duration of epilepsy before RF-TC of 9.5 years (1-38.6). Five patients had monthly seizures before RF-TC, 14 weekly, and 19 daily (for other clinical characteristics and RF-TC procedural details see Table 1).

Nineteen patients showed a clinical improvement after RF-TC. The median duration of improvement was 18.5 months (12-37) for the 4 improved patients with monthly seizure before RF-TC, 9 months (2-16) for the 5 improved patients with weekly seizures, and 11.5 months (1-48) for the 10 improved patients with daily seizures. In 8 patients the improvement was a complete seizure-freedom (42\%) and in 11 it was a reduction in seizures' frequency of at least 50\%. The remaining 19 patients did not show improvement in seizure frequency. None of our patients reported lasting adverse events: five patients had seizures during the RF-TC procedure, one patient reported headache after RF-TC and another one had transitory cerebral edema; eventually, 3 patients had a temporary increase in seizures’ frequency after RF-TC. 
After SEEG, 21 patients underwent surgical resection and among them, 11 had presented a clinical improvement after RF-TC. A median of 10 months passed from RF-TC to surgery (2-29). All 21 patients had a follow-up after surgery of at least 12 months: 16 showed a good surgical outcome (15 patients in Engel class I). Five patients did not show an important improvement after surgery (class III and IV of Engel). The median follow-up time available since RF-TC was 39 months (3-64). The median follow-up time available since surgery was 34 months (12-50).

\section{$\underline{\text { 3.2 Intra-individual statistics }}$}

An illustrative case of intra-individual statistics is depicted in figure 1. At an individual level, a reduction ( $\mathrm{p}<0.05$, surrogate analysis) of the assessed biomarkers (spikes, HFOs, and cross-rate) within the irritative, epileptogenic, and thermocoagulated zone was observed after RF-TC in a proportion of patients: in the irritative zone, 20 patients $(52 \%)$ showed a significant intra-individual reduction of spikes, 14 patients (36\%) of HFOs and $22(57 \%)$ of the cross-rate. A similar picture was observed in the EZ: a significant intra-individual decrease of biomarkers was observed for spikes in 20 patients (52\%), for HFOs in 13 patients (34\%), and for cross-rate in $22(57 \%)$. In the thermocoagulated zone, 18 patients (47\%) displayed a significant intra-individual reduction in spikes, $11(28 \%)$ in HFOs, and $24(63 \%)$ in cross-rate.

Results of surrogate analysis with regard to the clinical response after RF-TC are shown in Table 2. In EZ an intra-individual decrease in spikes was significantly more frequent in clinically improved patients than in non-improved ones $(15(78.9 \%)$ versus $5(26.3 \%), \mathrm{p}=0.002)$ and similarly for cross rates $(15(78.9 \%)$ vs $7(36.8 \%), p=0.02)$ while this difference was not significant for HFOs $(8$ $(42.1 \%)$ vs $5(26.3 \%)$ ) (assuming a false discovery rate of $15 \%)$. Within the thermocoagulated and irritative zones, we found a greater proportion of improved patients with an intra-individual reduction of the three biomarkers but without reaching significance, only in all domains combined an intraindividual decrease of cross rate was significantly more frequent in clinically improved patients than 
in non-improved ones $(14(73.7 \%)$ vs $4(21.1 \%)), p=0.02)$ (assuming a false discovery rate of $15 \%)$.

We also evaluated the results of surrogate analysis according to patient outcome after resective surgery. No significant differences in terms of frequency of an intra-individual decrease of biomarkers in the different zones were shown between the improved and non-improved patients after resective surgery (Table S1).

\subsection{Analysis at a group level}

\subsubsection{Modification of the rates of markers after RF-TC}

We first analyzed whether the RF-TC could reduce the rates of different markers in all the recorded channels, irrespective of the region of interest or the clinical outcome. In this case, comparing if the differences between post and pre values were lower than zero, we did not find a difference ( $p>0.15 / 0.6 / 0.25$ for spikes/HFOs/cross-rate).

Afterward, we tested whether the effect of RF-TC was region-specific by evaluating the irritative, the epileptogenic, and the thermocoagulated zone separately (Fig 2A). We observed a reduction in spikes $(\mathrm{p}=0.02)$, HFOs $(\mathrm{p}=0.03)$, and cross-rate $(\mathrm{p}=0.01)$ after RF-TC in the EZ. In the thermocoagulated zone, we observed a reduction of HFOs $(\mathrm{p}=0.007)$ and cross rate $(\mathrm{p}=0.005)$. In the irritative zone, spikes $(\mathrm{p}=0.03)$ and cross-rate $(\mathrm{p}=0.02)$ were reduced.

We also found a greater reduction of HFOs in thermocoagulated and epileptogenic zone (both with $\mathrm{p}=0.03$ ) in clinically improved patients compared to those with no clinical benefit (Fig 2B).

\subsubsection{Other factors associated with the modifications of marker rate}

In order to explore other possible predictors of markers' modifications after RF-TC, we included them in a multiple linear regression model with the following binary variables: the presence of SCIEDs on SEEG before RF-TC, the extent of irritative, epileptogenic, and thermocoagulated zones (regional vs extended), the etiology of epilepsy (both unknown vs known and due to MCD vs not due to MCD) and the topography of epilepsy (multilobar vs unilobar). In Fig 3A, we have plotted in gray 
the total variance of the different markers in the thermocoagulated zone (since this is the area with the most pronounced effects) that can be explained by each factor, and in black the variance explained solely by each factor that cannot be accounted by other variables.

We have then computed a new multiple linear regression, including the clinical response to RF-TC (bivariate variable) and only the two other factors, which were almost significant (extension of the irritative zone and SCIEDS). Both factors presented a significant association with the variation of almost all the markers, with the cross-rate as the marker with a better prediction (Fig 3B). In detail, significant regressions were found for the three markers (Spikes: $F(3,34)=4.81, p=0.0068, R^{2}=0.30$; HFOs: $F(3,34)=7.13, \mathrm{p}=0.0008, \mathrm{R}^{2}=0.39$; Cross-Rate: $\left.\mathrm{F}(3,34)=9.42, \mathrm{p}=0.0001, \mathrm{R}^{2}=0.45\right)$. Regarding spikes, the beta coefficient (i.e. the degree of changes in spike rate) for clinical response was equal to $-1.62(\mathrm{CI} 95 \%=[-3.81,0.95])$, the beta coefficient for SCIEDs was $-0.70(\mathrm{CI} 95 \%=[-$ $2.63,1.25])$ and for the extension irritative zone it was 2.56 ( CI 95\%=[0.45, 4.68]) (Fig 3B). For HFOs the beta coefficient for clinical response was equal to -7.01 (CI 95\%=[-14.51, 0.49]), for SCIEDs it was $-9.39($ CI 95\%=[-17.65, -1.15]) and for the extension of irritative zone it was 8.83 (CI $95 \%=[0.03,17.62])($ Fig 3B). For cross-rate the beta coefficient for clinical response was equal to $5.04(\mathrm{CI} 95 \%=[-10.67,0.58])$, for SCIEDs it was $-8.06(\mathrm{CI} 95 \%=[-14.19,-1.93])$ and for the extension of irritative zone it was 8.99 (CI 95\%=[2.31, 15.68]) (Fig 3B). SCIEDs and extension of irritative zone had a different effect on the variation of the markers: the presence of SCIEDs was associated with a reduction of the rate of HFOs and cross-rate after RF-TC, while an extended irritative zone implied a limited effect of RF-TC on the three marker rate (Fig 3C).

\subsubsection{Interictal marker changes and link with the outcome of resective surgery}

From a clinical point of view, a positive response to RF-TC was not associated with a subsequent successful outcome of resective surgery: of the 14 patients improved after surgery, only 9 had a favorable result after RF-TC, and 5 had not $(p=0.33)$. We, therefore, tested whether the effect of RFTC on the different markers could be a predictor of the success of the surgery independently of the clinical response to RF-TC. We compared the difference between post and pre values of the rates of 
markers in all channels in patients with a successful outcome of surgery versus those with a negative outcome, independently of any other factor. We found a significant decrease after RF-TC in all the markers in patients with a favorable surgery outcome $(\mathrm{p}=0.026$ for spikes, $\mathrm{p}=0.03$ for HFOs, $\mathrm{p}=0.03$ for cross-rate)(Fig. 4).

\section{DISCUSSION}

SEEG guided RF-TC is an emerging therapeutic method that allows the creation of small lesions in epileptogenic networks. However, the therapeutic effects after this procedure are uncertain and the predictive factors of success remain poorly known especially the potential electrophysiological predictors. ${ }^{1,6,7,9,10,11,27}$ In a previous case report, we showed a reduction of HFOs and spikes after RFTC, which corresponded to a good clinical outcome after this procedure. ${ }^{14}$ In this context, this study was conducted in order to estimate the effect of RF-TC on several interictal biomarkers of epileptogenicity measured from SEEG recordings. We concurrently analyzed the differences in the rates of both HFOs and spikes after RF-TC using an automatized quantified method that is supposed to better identify the electrophysiological markers compared to visual analysis. ${ }^{25,28}$ We demonstrate that RF-TC is able to modify spikes, HFOs, and cross-rate in SEEG traces and that there are correlations between these modifications and the clinical response to RF-TC.

Regarding spikes, we found a significant intra-individual reduction of spike rate in $52 \%$ of patients within the irritative and epileptogenic zones, and in $47 \%$ of patients within the thermocoagulated zone. At the group level, the spikes reduction was statistically significant in the epileptogenic and irritative zones. These results are consistent with previously published data. ${ }^{6,12,13}$ In one study, $31 \%$ of the patients had a more than $80 \%$ decrease of spike rates after RF-TC while spikes completely disappeared in $42 \%$ of patients. ${ }^{13}$ However, in the aforementioned papers, which were not based on an electrophysiological quantitative analysis, no significant association between spikes reduction and clinical effects of RF-TC was found. One research group reported a decrease of spikes in $25 \%$ of the clinically improved patients versus $33.3 \%$ in the non-responders but this difference was not significant. ${ }^{6}$ In our study instead, we could demonstrate at the intra-individual level that a reduction 
in spike rates within the EZ was significantly more frequent in patients with a clinical improvement after RF-TC.

With regard to HFOs, we observed at the group analysis a statistically significant reduction of their rate after RF-TC in thermocoagulated and epileptogenic zones. These results are in line with the previous works considering the role of HFOs as markers of epileptogenicity. ${ }^{14,15,29,30,31}$ We also showed a significantly greater reduction of HFOs in epileptogenic and thermocoagulated zone in patients with clinical improvement after RF-TC in comparison with non-improved patients.

In a previous work of our group, a combined measure of spikes and HFOs, called cross-rate, was shown to be a more sensitive marker of epileptogenicity than spikes or HFOs alone. ${ }^{16}$ In the present study, this marker was sensitive to RF-TC with a significant reduction of its rate at group analysis in the irritative, epileptogenic, and thermocoagulated zones. Moreover, as for spikes, we found an association between cross-rate reduction and clinical effects of RF-TC: at the intra-individual level, a reduction in cross-rate within the EZ was significantly more frequent in patients with a clinical improvement after RF-TC.

We observed slightly different results between the intra-individual and the group analysis, which could be linked to the different statistical methodologies applied in each analysis. For example, the fact that we did not reproduce a statistically significant reduction of HFOs in the majority of clinically improved patients in the intra-individual analysis can be explained by the insufficient number of HFOs in all areas for a proper surrogate analysis.

A decrease in seizure frequency of at least $50 \%$ during the first months following RF-TC was found to be a predictor of a good outcome after conventional cortectomy in a large series ${ }^{8}$ but this was not observed in others and in the present work. ${ }^{6}$ We observed a significant reduction of spikes, HFOs, and cross-rate after RF-TC in patients with a subsequent good outcome of resective surgery regardless of the clinical improvement after RF-TC. One possible explanation could be that RF-TC might contribute to the clinical success of resective surgery by limiting the primary epileptogenic network and the secondary epileptogenic foci. ${ }^{32,33}$ 
In our study, the presence of SCIEDs in SEEG traces before RF-TC was shown to be associated with the modifications of markers after RF-TC, in particular with a reduction of HFOs and cross-rate. Interestingly, a previous work has shown that SCIEDs could be predictive of a positive response to RF-TC. ${ }^{6}$ However, a clear causality between the reduction of the markers after RF-TC, the presence of SCIEDs, and the clinical improvement cannot be directly deduced because SCIEDs only partially explained the variation.

Another clinical-electrophysiological association derived from our results is that an extended irritative zone was a factor limiting the reduction of spikes, HFOs, and cross-rate after RF-TC. Other authors did not find any correlation between the extent of the irritative zone and the clinical effects of RF-TC. ${ }^{6}$ The extent of the irritative zone (spikes and HFOs) may reflect the size of epileptogenic networks in the brain, thus representing a limiting factor for the therapeutic effect of RF-TC. Again, a clear causal relationship between the SEEG-changes after RF-TC, the extent of the irritative zone, and the clinical response to RF-TC cannot be directly drawn.

\section{CONCLUSIONS}

In this work, we demonstrated changes in the rates of spikes, HFOs, and cross-rate after RF-TC with an automatized quantified method. The reduction of these electrophysiological markers appeared to be significantly related to both a good clinical response after RF-TC and to a favorable outcome of resective surgery. Future research is necessary to confirm these results. We suggest that a quantitative evaluation of the variation of these SEEG parameters after RF-TC may be a useful predictive tool of the clinical response of patients to this procedure. In the future, the practical implications of our finding in terms of the indication for repetitive RF-TC, modifications of drug therapy after RF-TC, and resective surgery need to be assessed. Our study focused on the short-term effect of RF-TC since we studied the immediate effects of the procedure. It is notable that the clinical effect of thermocoagulation is temporary in most of the cases and the long-term changes induced by RF-TC are unknown. 


\section{ACKNOWLEDGEMENTS}

We thank Dr. Rinki Singh from the Kings College Hospital of London for her comments on the manuscript and for the English revision. We thank Elodie Garnier data manager from Aix Marseille Univ, INSERM, INS, Inst Neurosci Syst, Marseille for her help with statistical revisions.

\section{DISCLOSURE OF CONFLICTS OF INTEREST}

M. Contento, F. Pizzo, V. J. López-Madrona, S. Lagarde, J. Makhalova, A. Trébuchon, S. M. Villalon, B. Giusiano, D. Scavarda, and R. Carron report no disclosures relevant to the manuscript. C.G. Bénar, N. Roehri, and F. Bartolomei have a pending patent (EP3422935A1) on the detection procedure licensed to MICROMED S.p. A.

\section{ETHICAL PUBLICATION STATEMENT}

We confirm that we have read the Journal's position on issues involved in ethical publication and affirm that this report is consistent with those guidelines.

\section{REFERENCES}

1. Catenoix $\mathrm{H}$, Bourdillon $\mathrm{P}$, Guénot $\mathrm{M}$, Isnard $\mathrm{J}$. The combination of stereo-EEG and radiofrequency ablation. Epilepsy Res 2018;142:117-120.

2. Guenot M, Isnard J, Catenoix H, Mauguière F, Sindou M. SEEG-guided RFthermocoagulation of epileptic foci : A therapeutic alternative for drug-resistant non-operable partial epilepsies. Adv Tech Stand Neurosurg. 2011;36:61-78.

3. Cossu M, Cardinale F, Casaceli G, Castana L, Consales A, D’Orio P et al. Stereo-EEG-guided radiofrequency thermocoagulations. Epilepsia. 2017;58:66-72.

4. Wang D, Wei P, Shan Y, Ren L, Wang Y, Zhao G. Optimized stereoelectroencephalographyguided radiofrequency thermocoagulation in the treatment of patients with focal epilepsy. Ann 
Transl Med. 2020;8:15-15.

5. Bourdillon P, Devaux B, Job-Chapron AS, Isnard J. SEEG-guided radiofrequency thermocoagulation. Neurophysiol Clin; 2018;48:59-64.

6. Dimova P, de Palma L, Job-Chapron AS, Minotti L, Hoffmann D, Kahane P. Radiofrequency thermocoagulation of the seizure-onset zone during stereoelectroencephalography. Epilepsia. 2017;58:381-392.

7. Cossu M, Fuschillo D, Casaceli G, Pelliccia V, Castana L, Mai R et al. Stereoelectroencephalography-guided radiofrequency thermocoagulation in the epileptogenic zone: A retrospective study on 89 cases. J Neurosurg. 2015;123:1358-1367.

8. Bourdillon $\mathrm{P}$, Isnard $\mathrm{J}$, Catenoix $\mathrm{H}$, Montavont $\mathrm{A}$, Rheims $\mathrm{S}$, Ryvlin $\mathrm{P}$ et al. Stereo electroencephalography-guided radiofrequency thermocoagulation (SEEG-guided RF-TC) in drug-resistant focal epilepsy: Results from a 10-year experience. Epilepsia. 2017;58:85-93.

9. Cossu M, Fuschillo D, Cardinale F, Castana L, Francione S, Nobili L et al. Stereo-EEGguided radio-frequency thermocoagulations of epileptogenic grey-matter nodular heterotopy. $\mathrm{J}$ Neurol Neurosurg Psychiatry. 2014;85:611-617.

10. Cossu M, Mirandola L, Tassi L. RF-ablation in periventricular heterotopia-related epilepsy. Epilepsy Res Epub 2017.:0-1.

11. Mirandola L, Mai RF, Francione S, Pelliccia V, Gozzo F, Sartori I et al. Stereo-EEG: Diagnostic and therapeutic tool for periventricular nodular heterotopia epilepsies. Epilepsia. 2017;58:1962-1971.

12. Catenoix H, Mauguière F, Montavont A, Ryvlin P, Guénot M, Isnard J. Seizures Outcome After Stereoelectroencephalography-Guided Thermocoagulations in Malformations of Cortical Development Poorly Accessible to Surgical Resection. Neurosurgery. 2015;77:9-14.

13. Catenoix H, Mauguière F, Guénot M, Ryvlin P, Bissery A, Sindou M et al. SEEG-guided 
thermocoagulations: A palliative treatment of nonoperable partial epilepsies. Neurology. 2008;71:1719-1726.

14. Scholly J, Pizzo F, Timofeev A, Valenti-Hirsch MP, Ollivier I, Proust F et al. High-frequency oscillations and spikes running down after SEEG-guided thermocoagulations in the epileptogenic network of periventricular nodular heterotopia. Epilepsy Res 2019;150:27-31.

15. Zijlmans M, Jacobs J, Zelmann R, Dubeau F, Gotman J. High-frequency oscillations mirror disease activity in patients with epilepsy. Neurology. 2009;72:979-986.

16. Roehri N, Pizzo F, Lagarde S, Lambert I, Nica A, McGonigal A et al. High-frequency oscillations are not better biomarkers of epileptogenic tissues than spikes. Ann Neurol 2018;83:84-97.

17. Isnard J, Taussig D, Bartolomei F, Bourdillion P, Catenoix H, Chassoux F et al. French guidelines on stereoelectroencephalography (SEEG). Neurophysiol Clin; 2018;48:5-13.

18. Engel J. Surgical Treatment of the Epilepsies. Raven Press; 1987.

19. Bancaud JT and J. Stereotaxic Approach to Epilepsy: Methodology of Anatomo-Functional Stereotaxic Investigations. Prog Neurol Surg. 1973;5:297-354.

20. Guenot M, Isnard J, Ryvlin P, Fischer C, Ostrowsky K, Mauguiere F et al. Neurophysiological monitoring for epilepsy surgery: The Talairach SEEG method - Indications, results, complications and therapeutic applications in a series of 100 consecutive cases. Stereotact Funct Neurosurg. 2002;77:29-32.

21. Medina Villalon S, Paz R, Roehri N, Lagarde S, Pizzo F, Colombet B et al. EpiTools, A software suite for presurgical brain mapping in epilepsy: Intracerebral EEG. J Neurosci Methods; 2018;303:7-15. Available at: http://meg.univ-amu.fr/wiki/GARDEL:presentation

22. Bartolomei F, Chauvel P, Wendling F. Epileptogenicity of brain structures in human temporal lobe epilepsy: A quantified study from intracerebral EEG. Brain. 2008;131:1818-1830. 
23. Bartolomei F, Nica A, Valenti-Hirsch MP, Adam C, Denuelle M. Interpretation of SEEG recordings. Neurophysiol Clin; 2018;48:53-57.

24. Colombet B, Woodman M, Badier JM, Bénar CG. AnyWave: A cross-platform and modular software for visualizing and processing electrophysiological signals. J Neurosci Methods 2015;242:118-126.

25. Roehri N, Lina JM, Mosher JC, Bartolomei F, Benar CG. Time-Frequency Strategies for Increasing High-Frequency Oscillation Detectability in Intracerebral EEG. IEEE Trans Biomed Eng. 2016;63:2595-2606. Available at: https://meg.univ-amu.fr/wiki/AnyWave:Plug$\underline{\text { ins }}$

26. Cohen MX. Nonparametric permutation testing: From Analyzing neural time series data: theory and practice. 2014.

27. Bourdillon P, Cucherat M, Isnard J, Ostrowsky-Coste K, Catenoix H, Guenot M et al. Stereoelectroencephalography-guided radiofrequency thermocoagulation in patients with focal epilepsy: A systematic review and meta-analysis. Epilepsia. 2018;59:2296-2304.

28. Navarrete M, Alvarado-Rojas C, Le Van Quyen M, Valderrama M. RIPPLELAB: A comprehensive application for the detection, analysis and classification of high frequency oscillations in electroencephalographic signals. PLoS One. 2016;11(6): e0158276.

29. Jacobs J, Zijlmans M, Zelmann R, Chatillon CE, Hall J, Olivier A et al. High-frequency electroencephalographic oscillations correlate with outcome of epilepsy surgery. Ann Neurol. 2010;67:209-220.

30. Jacobs J, Wu JY, Perucca P, Zelmann R, Mader M, Dubeau F et al. Removing high-frequency oscillations. Neurology Epub 2018;00:e1-e13.

31. Höller Y, Kutil R, Klaffenböck L, Thomschewski A, Höller P M, Bathke A C et al. Highfrequency oscillations in epilepsy and surgical outcome. A meta-analysis. Front Hum 
Neurosci. 2015;9:1-14.

32. Morell F, deToledo-Morell L. From mirror focus to secondary epileptogenesis in man: an historical review. Adv. Neurol. 1999;81:11-23.

33. Geller AS, Friedman D, Fang M, Doyle WK, Devinsky O, Dugan P. Running-down phenomenon captured with chronic electrocorticography. Epilepsia Open 2018;3(4):528-534. 


\section{FIGURE LEGENDS}

Figure 1. An example of the surrogate analysis for spikes, HFOs, and cross-rate and a graphical representation of changes in the rates of the three epileptogenic biomarkers after RF-TC (patient 6).

Surrogate distributions for spikes (A), HFOs (B), and cross-rate (C) in the thermocoagulated area of patient 6 (female, clinically improved after RF-TC and seizure-free after resective surgery). These distributions were obtained through 5000 permutations of events between conditions. The cross in each graph displayed the original value of the difference between the rate of each marker after and before RF-TC: in these three graphs, the three original values fall outside the intervals of the variability given by the surrogate analysis, meaning that RF-TC confers a statistically significant reduction in this area for these three markers (see the text for the details). D;E;F Representation of the difference between post and pre values of the marker rates (rate after RF-TC - rate before RFTC) on the 3D SEEG implantation scheme in patient 6 . In the figure, the difference between post and pre values of spikes $(\mathbf{D})$, HFOs $(\mathbf{E})$, and cross-rate $(\mathbf{F})$ are respectively shown. The values of these differences are displayed by the color bar and the size of the spheres (the bigger the sphere, the higher is the difference between post and pre absolute values). G; H SEEG traces before (G) and after RF-TC $(\mathbf{H})$ : the reduction of the interictal activity and of its amplitude can be clearly seen. Among the displayed channels, note that DYS 1-2/2-3/3-4 and $\mathrm{H}$ 3-4/4-5 correspond to the thermocoagulated zone. The spikes on the displayed SEEG traces are marked by Delphos (grey rectangles $) . \mathrm{TH}=$ thermocoagulated; HFOs $=$ High Frequency Oscillations; $\mathrm{CR}=$ Cross rate; RF$\mathrm{TC}=$ radiofrequency thermocoagulation.

Figure 2. Group analysis of the modification of the rates of the three epileptogenic biomarkers after RF-TC.

A. Comparison between the difference between post and pre values for all markers against zero in the different areas $(* / * * \mathrm{p}<0.05 / 0.01, \mathrm{t}$-test $)$. Significant negative values indicate a decrease of the 
biomarker after RF-TC. For all figures, the central mark of the box indicates the median, and the bottom and top edges of the box indicate the 25 th and 75 th percentiles, respectively. The whiskers extend to the most extreme data points not considered outliers. B. Effect of clinical response to RFTC on the different markers after thermocoagulation in the different areas. Comparison between the difference between post and pre values in clinically improved versus no improved patients $(* / * * \mathrm{p}<$ 0.05/0.01, $\mathrm{F}(1,108)=11.62 \quad / \quad \mathrm{F}(1,108)=11.96 \quad / \quad \mathrm{F}(1,108)=11.98 \quad$ for thermocoagulated/irritative/epileptogenic zone, respectively). Significant values indicate that biomarkers have a more pronounced decrease in clinically improved patients. Bars represent the mean \pm standard error of the mean (s.e.m.) between patients. Stars represent the p-value. No impr $=$ not clinically improved after RF-TC; Impr = clinically improved after RF-TC; HFOs = HighFrequency Oscillations.

\section{Figure 3. Analysis of the factors associated with the modifications of marker rate after RF-TC.}

A. Multiple linear regression model with 7 possible clinical predictors of the modifications of the rates of the markers after RF-TC in the thermocoagulated zone. Gray bars represent the total variance that can be explained by each factor, while black bars are the variance explained solely by each factor that cannot be accounted by other variables. HFOs = High Frequency Oscillations; SCIEDs = sub-continuous interictal epileptiform discharges; $\mathrm{IR}=$ irritative zone; $\mathrm{EZ}=$ epileptogenic zone; $\mathrm{TH}=$ thermocoagulated zone; eti unknown = unknown etiology; eti mcd = etiology due to a malformation of cortical development; topo epi = topography of epilepsy. Stars represent significant p-values, indicating that the plotted variable independently affects the pre- and post-RF-TC rate of the biomarker $(* / * * \mathrm{p}<0.05 / 0.01)$.

B. Multiple linear regression model with sub-continuous interictal epileptiform discharges (SCIEDs) and extension of the irritative zone (IR) as secondary predictors of modifications of the rates of markers combined with the clinical response $(* / * * p<0.05 / 0.01)$. Clin resp. $=$ clinical response to RFTC. C. Difference of markers rate after RF-TC in function of the sub-continuos interictal 
epileptiform discharges (left) and of the extension of the irritative zone (right). Bars represent the mean \pm s.e.m. between patients.

\section{Figure 4: Interictal marker changes after RF-TC and outcome of resective surgery.}

Comparison between the difference between post and pre delta values (rate post-RF-TC - rate preRF-TC) of the different markers in all channels in patients with a successful outcome of surgery versus those with a negative outcome. Stars indicate a more pronounced decrease of biomarkers in patients with a successful outcome $(* \mathrm{p}<0.05 \mathrm{~F}(1,57)=19.25)$. 


\section{TABLES}

\section{$\underline{\text { Table } 1}$}

Population's clinical features and characteristics regarding the SEEG recordings, the RF-TC procedures, and the clinical response to RF-TC.

\begin{tabular}{|c|c|c|c|}
\hline Variables & $\mathbf{N}=\mathbf{3 8}$ & Range & Median \\
\hline Febrile seizures during childhood & 4 & & \\
\hline Familiar history of epilepsy & 5 & & \\
\hline History of perinatal sufferance & 6 & & \\
\hline History of cranial trauma & 3 & & \\
\hline History of CNS infection & 2 & & \\
\hline Delay in development & 11 & & \\
\hline Number of AEDs tried before RF-TC & & $2-10$ & 7 \\
\hline \multicolumn{4}{|l|}{ Etiology of epilepsy } \\
\hline$M C D^{*}$ & 12 & & \\
\hline Polymicrogyria & 1 & & \\
\hline$F C D^{* *}$ & 6 & & \\
\hline$N D T^{* * *}$ & 2 & & \\
\hline$H S^{* *}$ & 3 & & \\
\hline$C T$ & 1 & & \\
\hline$C V D$ & 2 & & \\
\hline Unspecified histology & 5 & & \\
\hline Normal MRI & 6 & & \\
\hline \multicolumn{4}{|l|}{ Lateralization of epilepsy } \\
\hline Right-sided & 16 & & \\
\hline Left-sided & 18 & & \\
\hline Bilateral & 4 & & \\
\hline
\end{tabular}

Topography of epilepsy 


\begin{tabular}{|c|c|c|c|}
\hline Temporal & 13 & & \\
\hline Parietal/Occipital & 5 & & \\
\hline Frontal & 5 & & \\
\hline Insulo-opercular & 3 & & \\
\hline Multilobar & 12 & & \\
\hline \multicolumn{4}{|l|}{ Lateralization of electrodes implantation } \\
\hline Right-sided & 3 & & \\
\hline Left-sided & 5 & & \\
\hline Bilateral with left hemispheric predominance & 18 & & \\
\hline Bilateral with right hemispheric predominance & 12 & & \\
\hline Total number of contacts & & $47-142$ & 84.5 \\
\hline Total number of contacts in EZ & & $4-59$ & 16 \\
\hline Number of contacts thermocoagulated & & $2-28$ & $10.5(11.6 \%)$ \\
\hline Clinically improved patients & & $3-28$ & $9(11.1 \%)$ \\
\hline Not Clinically improved patients & & $2-22$ & $13(13.5 \%)$ \\
\hline Number of contacts thermocoagulated in EZ & & $2-25$ & $8(60.7 \%)$ \\
\hline Clinically improved patients & & $3-25$ & $8(50 \%)$ \\
\hline Not Clinically improved patients & & $2-22$ & $8(63.6 \%)$ \\
\hline
\end{tabular}

$\mathrm{RF}-\mathrm{TC}=$ radiofrequency thermocoagulation; $\mathrm{AEDs}=$ antiepileptic drugs $; \mathrm{MCD}=$ malformations of cortical development FCD = focal cortical dysplasia; NDT = neurodevelopmental tumors; HS = hippocampal sclerosis; $\mathrm{CT}=$ cranial trauma $\mathrm{CVD}=$ cerebrovascular diseases $; \mathrm{EZ}=$ epileptogenic zone. * not more definable MCD. ** confirmed by the histology after the resective surgery *** NDT confirmed by the histology after resective surgery (one low grade dysembryoplastic neuroepithelial tumor (DNET) and one angiocentric neuroepithelial tumor (ANET). 


\section{Table 2.}

Surrogate analysis according to the presence of clinical improvement after RF-TC for all the patients who showed a significant intra-individual reduction of the different markers in the three areas. In the table are also reported the p-value resulting from the Fisher's Exact Tests performed between clinically improved and not clinically improved patients for each marker in each area.

\begin{tabular}{lccc}
\hline \multicolumn{1}{c}{ Analyzed Zone } & $\begin{array}{c}\text { Spikes } \\
\text { reduction }\end{array}$ & $\begin{array}{c}\text { HFOs } \\
\text { reduction }\end{array}$ & $\begin{array}{c}\text { Cross Rate } \\
\text { reduction }\end{array}$ \\
\hline Irritative Zone & $13(68.4 \%)$ & $8(42.1 \%)$ & $13(68.4 \%)$ \\
Clinically improved patients & $7(36.8 \%)$ & $6(31.6 \%)$ & $9(47.4 \%)$ \\
Not clinically improved patients & $\mathrm{p}=0.10$ & $\mathrm{p}=0.73$ & $\mathrm{p}=0.32$ \\
& & & \\
\hline
\end{tabular}

Epileptogenic Zone

$\begin{array}{llll}\text { Clinically improved patients } & 15(78.9 \%) & 8(42.1 \%) & 15(78.9 \%)\end{array}$

$\begin{array}{lll}\text { Not clinically improved patients } & 5(26.3 \%) & 5(26.3 \%)\end{array}$

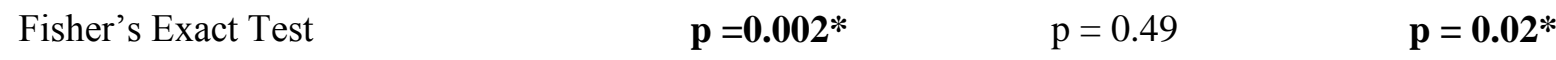

Thermocoagulated Zone

Clinically improved patients

$12(63.2 \%)$

$7(36.8 \%)$

$14(73.7 \%)$

Not clinically improved patients

$6(31.6 \%)$

$4(21.1 \%)$

$10(52.6 \%)$

Fisher's Exact Test

$\mathrm{p}=0.10$

$p=0.47$

$\mathrm{p}=0.31$

All areas all together at the same

time

Clinically improved patients

$10(52.6 \%)$

$3(15.8 \%)$

$14(73.7 \%)$ 


\begin{tabular}{lccc}
\hline Not clinically improved patients & $4(21.1 \%)$ & 0 & $4(21.1 \%)$ \\
Fisher's Exact Test & $\mathrm{p}=0.30$ & $\mathrm{p}=0.24$ & $\mathbf{p}=\mathbf{0 . 0 2} *$ \\
\end{tabular}

* statistically significant $\mathrm{p}$ values. HFOs $=$ High-frequency oscillations.

\section{FIG1}

A

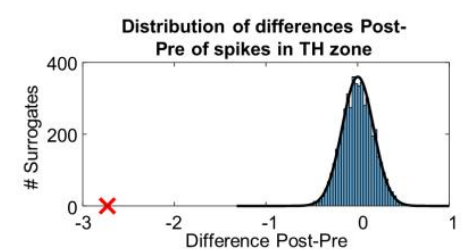

D

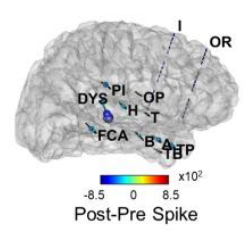

G

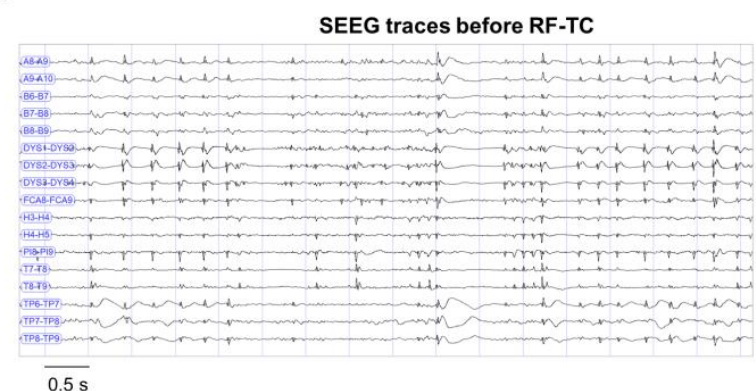

B

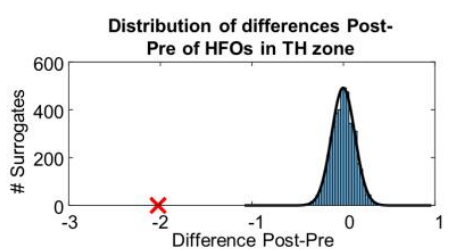

E

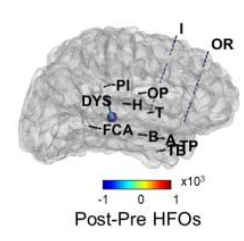

$\mathrm{H}$ c

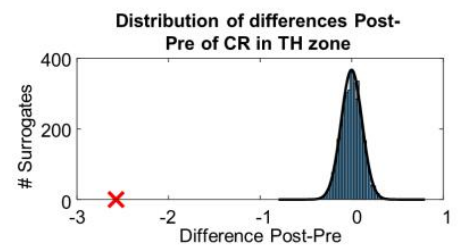

F

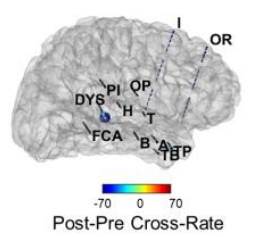

SEEG traces after RF-TC

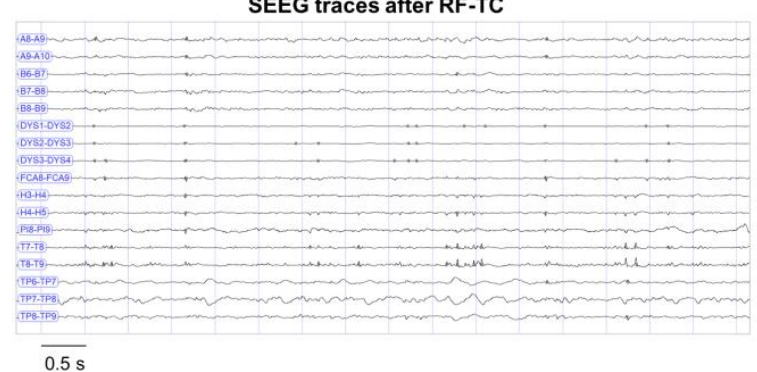




$$
\begin{array}{ll}
\quad & \text { Thermocoagulated zone (TH) } \\
\text { A Irritative zone (IR) } \\
\text { Epileptogenic zone (EZ) }
\end{array}
$$
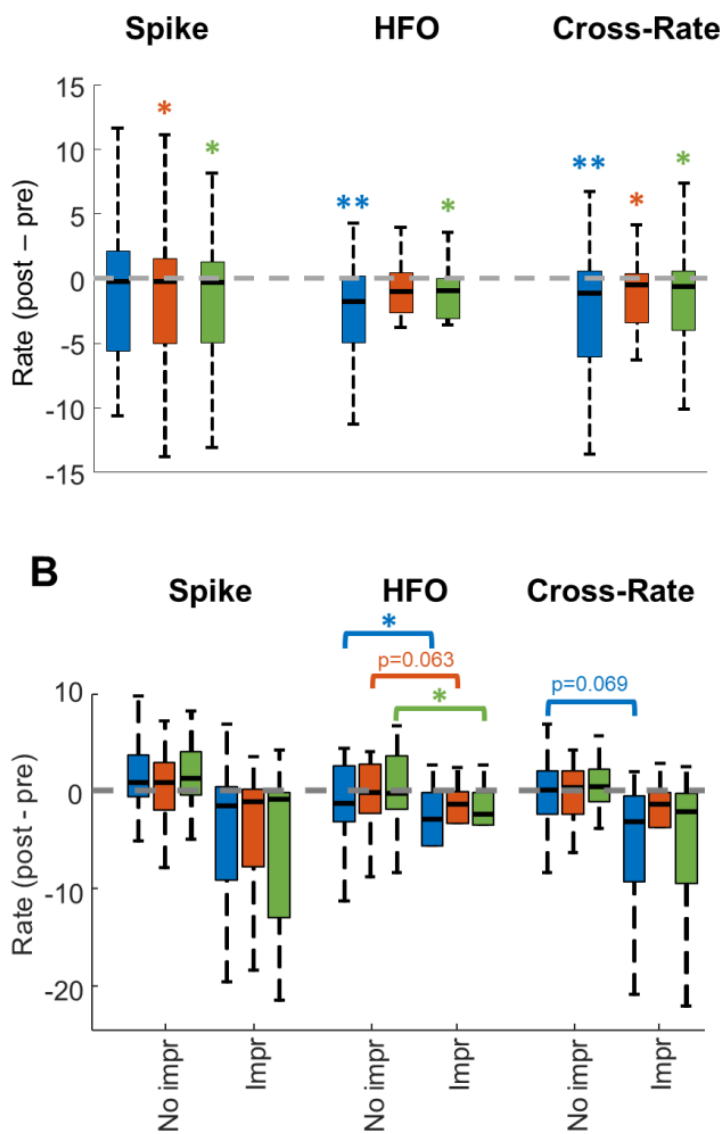

FIG2 
A

B
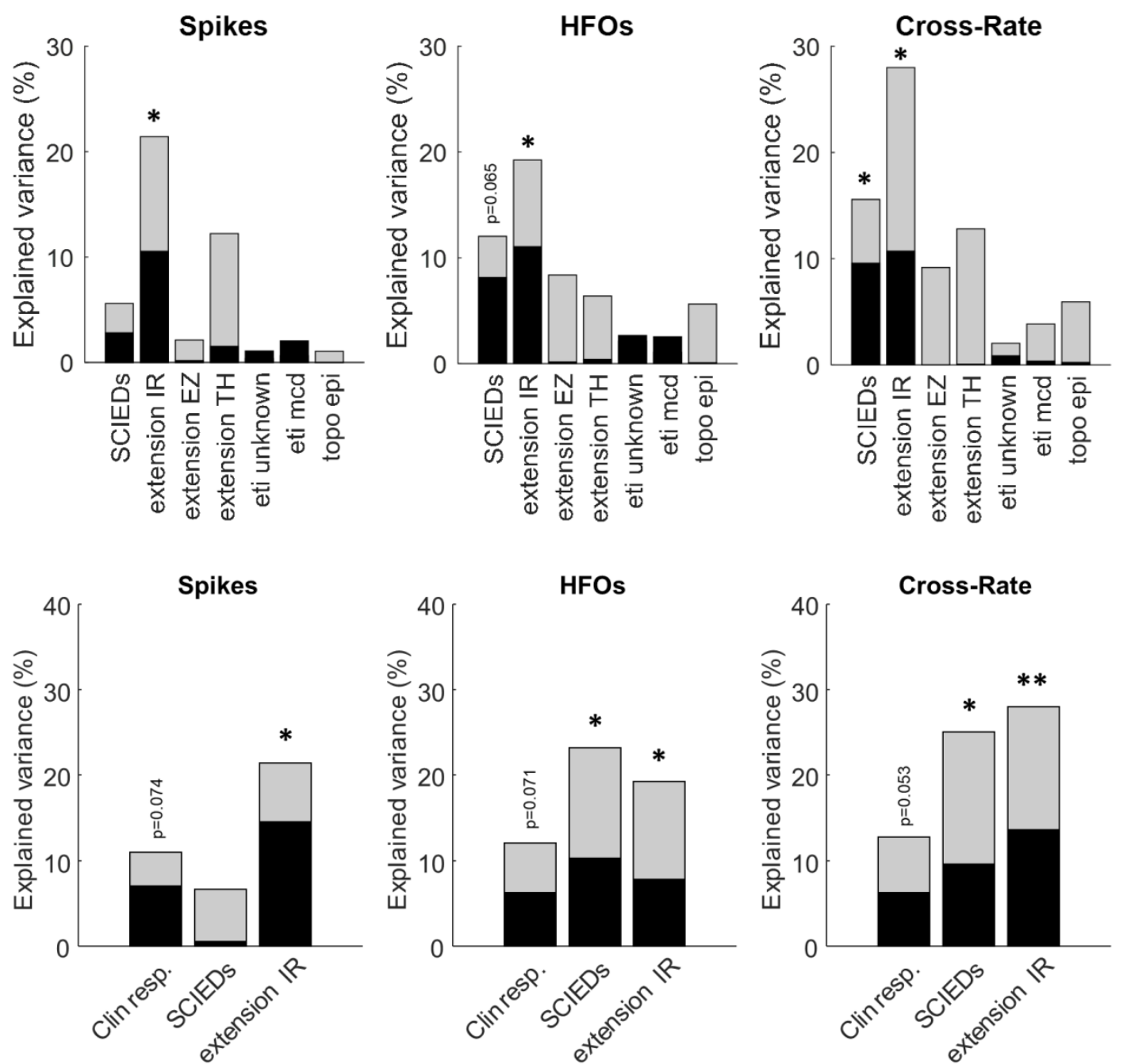

C

$\square 7$ Absence of subcontinuous interictal activity

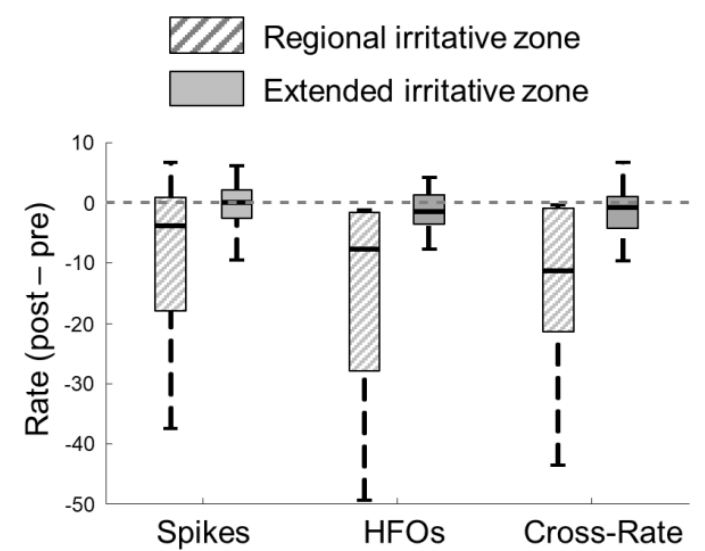

FIG3 


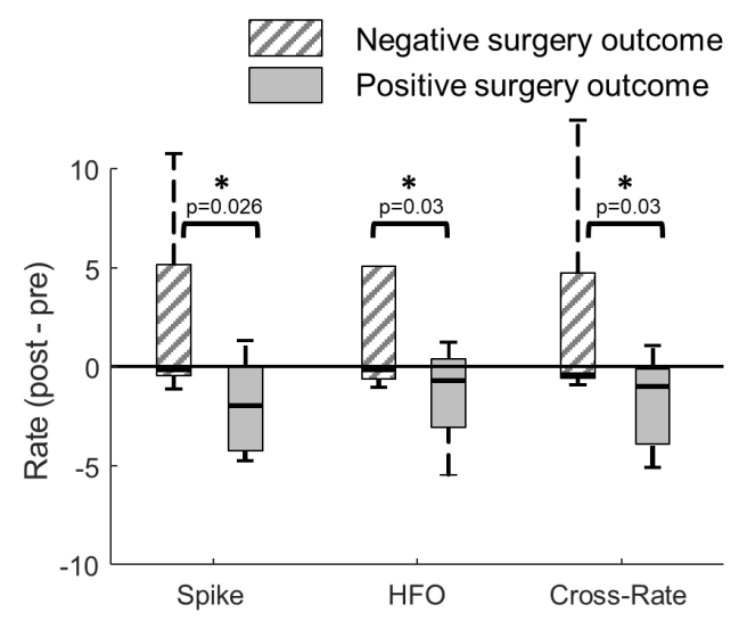

FIG4 